\title{
Global synchronization analysis for complex networks with time varying coupling delay
}

\author{
Jinfang Zhang ${ }^{1}$ Yuanhua Qiao $^{1 *}$ Xianghui Chen ${ }^{2}$ Xiaojie Liu ${ }^{3}$ \\ ${ }^{1}$ College of Applied Sciences, Beijing University of Technology, Beijing 100124, China \\ ${ }^{2}$ College of Applied Sciences, Beijing University of Technology, Beijing 100124, China \\ ${ }^{3}$ College of Applied Sciences, Beijing University of Technology, Beijing 100124, China
}

\begin{abstract}
:
Global synchronization analysis for complex networks with time varying coupling delay is investigated in this paper. The delay considered in this paper is time-varying. Sufficient conditions depending on the time delay are given based on Lyapunov functional, linear matrix inequality and Kronecker product technique. We find that the conditions are dependent on the bounds of both time-delay and its derivative, and the derivative of the time-varying delay can be any value in the bounds. Finally, a numerical example is given to validate the effectiveness of the obtained results.
\end{abstract}

Key words: Complex networks; Global synchronization; Linear matrix inequality; Kronecker product.

\section{Introduction}

Dynamical recurrent neural networks are used extensively in classification of patterns, associative memories and optimization [1-2] etc. In these networks, there also exist more complicated dynamics, therefore, the theory of complex networks has become a focal research topic and attracted a great deal of attention in various fields. Recent years have witnessed an increasing interest in complex networks from the science and technology communities [3-4].

Recently, it is found that synchronization is one of the most important dynamical properties of complex networks and has been extensively investigated in different ways [5-10]. In [5], L $\ddot{u}$ and Chen considered a dynamical network and gave the conditions for local synchronization. Because of the network traffic congestions as well as the finite speed of signal transmission over the links, time delays occur commonly in complex networks. Therefore, Li, and Chen [6] improved the models with no delays, the case with constant coupling delays is considered, and they also analyzed the Synchronization problem [7]. However, in many real-world networks time delay is varying, therefore time varying coupling delays are considered in [8-9]. By using free-weighting matrices, some synchronization criteria for general complex dynamical networks with time-varying delays are proposed. However, the computation is huge, and there are a large amount of variables in the condition. Moreover, in order to derive the synchronization condition, in some papers [6-9], the time-varying delay $\tau(t)$ is usually confined to $0 \leq \tau(t) \leq \tau$ (lower bound of the delay is zero.), and the derivative is restricted less than 1. Therefore, how to improve the system performance by removing the redundant variables and reducing computation still remain unsolved. Motivated by the mentioned discussions, we study the synchronization problems for general complex networks with time varying delay. Using different Lyapunov functionals given, the synchronization criteria derived turn out to be less conservative.

The remainder of this paper is organized as follows: in Section 2, the investigated system is formulated and some lemmas and notations are given. In Section 3, the conditions for synchronization are derived. In Section 4, a numerical example is presented to demonstrate the effectiveness and the advantage of the proposed method. Finally, conclusions are drawn in Section 5.

\section{Problem formulations}

Suppose the nodes are coupled with states $x_{i}(t)$, $i \in\{1, \cdots N\}$, then the dynamic neural networks (DNNs) of general form can be described by

$$
\begin{aligned}
& \dot{x}_{i}(t)=-C x_{i}(t)+D f\left(x_{i}(t)\right)+B f\left(x_{i}(t-\tau(t))\right)+I(t)+c \sum_{j=1 j \neq i}^{N} G_{i j} A\left[x_{j}(t-\tau(t))-\right. \\
& \left.x_{i}(t-\tau(t))\right]+c \sum_{j=1 j \neq i}^{N} G_{i j} A_{\tau}\left[x_{j}(t-\tau(t))-x_{i}(t)\right]
\end{aligned}
$$


in which $x_{i}(t)=\left[x_{i_{1}}(t), x_{i_{2}}(t), \cdots, x_{i_{n}}(t)\right]^{T} \in R^{n}$ is the state vector of the $i$-th network at time $t$. The functions $f\left(x_{i}(t)\right)=\left[f_{1}\left(x_{i 1}(t)\right), f_{2}\left(x_{i 2}(t)\right), \cdots, f_{n}\left(x_{i_{n}}(t)\right)\right]^{T}$ is sufficiently smooth nonlinear vector fields, and $I(t)=\left[I_{1}(t), I_{2}(t), \cdots, I_{n}(t)\right]^{T} \in R^{n}$ is the external input vector; $C=\operatorname{diag}\left\{c_{1}, c_{2}, \cdots, c_{n}\right\}>0, D=\left[d_{i j}\right]_{n \times n}, \quad B=\left[b_{i j}\right]_{n \times n}$ here $A=\left[a_{i j}\right]_{n \times n}$ and $A_{\tau}=\left[a_{i j}^{\prime}\right]_{n \times n}$ are inner-coupling matrix, $G=\left[G_{i j}\right]_{N \times N}$ represent the outer-coupling connections. The constant $c>0$ represents the coupling strength, $\tau(t)$ represents the time constant delay.

For the network (1), the following assumptions are needed.

Assumption (H1) $\tau(t)$ is the interval time-varying delay satisfying

$0 \leq h_{1} \leq \tau(t) \leq h_{2}, \quad \dot{\tau}(t) \leq \mu<+\infty$ and $h_{21}=h_{2}-h_{1}$.

Assumption (H2) The outer-coupling configuration matrices of the complex networks satisfy:

$$
\left\{\begin{array}{c}
G_{i j}=G_{j i} \geq 0, i \neq j, \\
G_{i i}=-\sum_{j=1, j \neq i}^{N} G_{i j}, i, j=1,2, \cdots, N .
\end{array}\right.
$$

Assumption (H3) For any constants $\sigma_{i}^{-}, \sigma_{i}^{+}$, the functions satisfy:

$\sigma_{i}^{-} \leq \frac{f_{i}\left(x_{1}\right)-f_{i}\left(x_{2}\right)}{x_{1}-x_{2}} \leq \sigma_{i}^{+}, \quad i=1,2, \cdots n$

we denote

$\Lambda_{1}=\operatorname{diag}\left(\sigma_{1}^{+} \sigma_{1}^{-}, \sigma_{2}^{+} \sigma_{2}^{-}, \cdots, \sigma_{n}^{+} \sigma_{n}^{-}\right)$,

$\Lambda_{2}=\operatorname{diag}\left(\frac{\sigma_{1}^{+}+\sigma_{1}^{-}}{2}, \frac{\sigma_{2}^{+}+\sigma_{2}^{-}}{2}, \cdots, \frac{\sigma_{n}^{+}+\sigma_{n}^{-}}{2}\right)$

Based on Assumption (H2), system (1) can be rewritten as the following form:

$\dot{x}_{i}(t)=-C x_{i}(t)+D f\left(x_{i}(t)\right)+B f\left(x_{i}(t-\tau(t))\right)+I(t)+c \sum_{j=1}^{N} G_{i j} A x_{j}(t-$

$\tau(t))+c \sum_{j=1}^{N} G_{i j} A_{\tau} x_{j}(t-\tau(t))-c G_{i i} A_{\tau}\left[x_{i}(t-\tau(t))-x_{i}(t)\right]$

The initial conditions of (2) are given by $x_{i}(s)=\phi_{i}(s) . s \in\left[t_{0}-h_{2}, t_{0}\right], i=1,2, \cdots N$, where $\phi_{i}(\cdot)=\left[\phi_{i 1}(\cdot), \phi_{i 2}(\cdot), \cdots \phi_{i n}(\cdot)\right]^{T} \in C\left(\left[t_{0}-h_{2}, t_{0}\right], R^{n}\right)$ and in order to simplify we set $G_{i i}=l$.

Remark 1: The constants $\sigma_{i}^{-}, \sigma_{i}^{+}$in assumption $\mathrm{H} 3$ are allowed to be any value. Then, most of the previous results in similar networks are just special cases of this assumption, which means that the activation functions are more general than that of other work.

Combining with the sign of Kronecker product, model (2) can be rewritten as:

$$
\begin{aligned}
& \dot{x}(t)=-\left(I_{N} \otimes C\right) x(t)+\left(I_{N} \otimes D\right) F(x(t))+\left(I_{N} \otimes B\right) \\
& F(x(t-\tau(t)))+I^{\prime}(t)+c\left(G \otimes\left(A+A_{\tau}\right)\right) x(t-\tau(t)) \\
& +c l\left(I_{N} \otimes A_{\tau}\right)[x(t-\tau(t))-x(t)]
\end{aligned}
$$

With $x(t)=\left[x_{1}^{T}(t), x_{2}^{T}(t), \cdots x_{N}^{T}(t)\right]^{T} F(x(t))=\left[f^{T}\left(x_{1}(t)\right), f^{T}\left(x_{2}(t)\right)\right.$, $\left.\cdots, f^{T}\left(x_{N}(t)\right)\right]^{T}$ and $I^{\prime}(t)=\left[I^{T}(t), I^{T}(t), \cdots, I^{T}(t)\right]^{T}$.

Definition1 [10]: Dynamical networks (3) is said to be global exponential synchronization, if for any initial conditions $\quad \phi_{i}(s), \phi_{j}(s) \in C\left(\left[t_{0}-h_{2}, t_{0}\right], R^{n}\right)$ $(i, j=1,2, \cdots N)$.There exist $T>t_{0} \quad$ and $\quad \varepsilon>0 \quad$ such that $\left\|x_{i}(t)-x_{j}(t)\right\| \leq M e^{-\varepsilon t}$, in which $t>T$ and $\|$. denotes the Euclidean norm.

Lemma 1 Let $\otimes$ denote the notation of Kronecker product. Then, the following relationships hold

(1) $(\alpha A) \otimes B=A \otimes(\alpha B)$

(2) $(A+B) \otimes C=A \otimes C+B \otimes C$

(3) $(A \otimes B)(C \otimes D)=(A C) \otimes B D$

Lemma $2[11]$ Let $e=(1,1, \cdots, 1)^{T}, E_{N}=e e^{T}$, $U=N I_{N}-E_{N}, P \in R^{n \times n}, x=\left(x_{1}^{T}, x_{2}^{T} \cdots x_{N}^{T}\right)^{T}$, $y=\left(y_{1}^{T}, y_{2}^{T} \cdots y_{N}^{T}\right)^{T}$ with $x_{k}, y_{k} \in R^{n},(k=1,2, \cdots N)$, then $x^{T}(U \otimes P) y=\sum_{1 \leq i<j \leq N}^{N}\left(x_{i}-x_{j}\right)^{T} P\left(y_{i}-y_{j}\right)$

Lemma 3[12] For any constant matrix $X \in R^{n \times n}$, $X=X^{T} \geq 0$, a scalar functional $h:=h(t) \geq 0$, and a vector function $\dot{x}:[-h, 0] \rightarrow R^{n}$, the following inequality hold 
$-h \int_{t-h}^{t} \dot{x}(s)^{T} X \dot{x}(s) d s \leq-[x(t)-x(t-h)]^{T} X[x(t)-x(t-h)]$

\section{MAIN RESULTS}

In this the section, by utilizing the improved techniques used in [13], we obtain the following global exponential synchronization criterion for system (3).

Theorem 1: For given c, $\tau(t)>0$, and supposing that Assumptions (H1)-(H3) hold, if there exist $P=P^{T}>0$, $Q_{i}=Q_{i}{ }^{T}>0, \quad R_{i}=R_{i}{ }^{T}>0, \quad(i=1,2,3) \quad$ and $Z_{j}=Z_{j}{ }^{T}>0(j=1,2)$, positive diagonal matrices $H, K, W, V$, such that the following LMIS hold for all $1 \leq i<j \leq N$

$\Phi_{i j}^{1}=\Phi_{i j}-\left[\begin{array}{llll}0_{n, 3 n} & -I_{n} & I_{n} & 0_{n, 3 n}\end{array}\right]^{T} Z_{2}\left[\begin{array}{llll}0_{n, 3 n} & -I_{n} & I_{n} & 0_{n, 3 n}\end{array}\right]<0$

$\Phi^{2} i j=\Phi_{i j}-\left[\begin{array}{lllll}0_{n, 3 n} & I_{n} & 0_{n} & -I_{n} & 0_{n, 2 n}\end{array}\right]^{T} Z_{2}\left[\begin{array}{lllll}0_{n, 3 n} & I_{n} & 0_{n} & -I_{n} & 0_{n, 2 n}\end{array}\right]<0$

Where $\Phi_{i j}=\left[\begin{array}{ccccc}\Omega_{11} & \Omega_{12} & \Omega_{13} & \Omega_{14} & Z_{1} \\ * & \Omega_{22} & \Omega_{23} & \Omega_{24} & 0 \\ * & * & \Omega_{33} & \Omega_{34} & 0 \\ * & * & * & \Omega_{44} & Z_{2} \\ * & * & * & * & \Omega_{55} \\ * & * & * & * & * \\ * & * & * & * & * \\ * & * & * & * & *\end{array}\right.$

$\left.\begin{array}{ccc}0 & 0 & 0 \\ 0 & 0 & 0 \\ 0 & 0 & 0 \\ Z_{2} & 0 & 0 \\ 0 & W \Lambda_{2} & 0 \\ -Q_{2}-Z_{2}-V \Lambda_{1} & 0 & V \Lambda_{2} \\ * & -R_{1}-W & 0 \\ * & * & -R_{2}-V\end{array}\right]$

with

$\Omega_{11}=-P C-C^{T} P+\sum_{i=1}^{3} Q_{i}-c l P A_{\tau}-c l A_{\tau}{ }^{T} P-Z_{1}+C^{T}$

$\left(h_{1}{ }^{2} Z_{1}+h_{12}{ }^{2} Z_{2}\right)\left(C+c l A_{\tau}\right)+c^{2} l^{2} A_{\tau}{ }^{T}\left(h_{1}{ }^{2} Z_{1}+h_{12}{ }^{2} Z_{2}\right)$ $A_{\tau}+\operatorname{clC} C^{T}\left(h_{1}{ }^{2} Z_{1}+h_{12}{ }^{2} Z_{2}\right) A_{\tau}-H \Lambda_{1}$

$\Omega_{12}=-C^{T}\left(h_{1}^{2} Z_{1}+h_{12}^{2} Z_{2}\right) D+P D-c l A_{\tau}^{T}\left(h_{1}^{2} Z_{1}\right.$ $\left.+h_{12}{ }^{2} Z_{2}\right) D+H \Lambda_{2}$
$\Omega_{13}=-C^{T}\left(h_{1}{ }^{2} Z_{1}+h_{12}{ }^{2} Z_{2}\right) B-c l A_{\tau}^{T}\left(h_{1}{ }^{2} Z_{1}\right.$

$\left.+h_{12}{ }^{2} Z_{2}\right) B+P B$

$\Omega_{14}=C^{T}\left(h_{1}{ }^{2} Z_{1}+h_{12}{ }^{2} Z_{2}\right)\left(c N G_{i j}\left(A+A_{\tau}\right)-c l A_{\tau}\right)$

$-c N G_{i j} P\left(A+A_{\tau}\right)+c l P A_{\tau}-c^{2} l N G_{i j} A_{\tau}^{T}\left(h_{1}^{2} Z_{1}\right.$

$\left.+h_{12}{ }^{2} Z_{2}\right)\left(A_{\tau}+A\right)-c^{2} l^{2} A_{\tau}{ }^{T}\left(h_{1}{ }^{2} Z_{1}+h_{12}{ }^{2} Z_{2}\right) A_{\tau}$

$\Omega_{22}=D^{T}\left(h_{1}^{2} Z_{1}+h_{12}{ }^{2} Z_{2}\right) D+\sum_{i=1}^{3} R_{i}-H$

$\Omega_{23}=D^{T}\left(h_{1}{ }^{2} Z_{1}+h_{12}{ }^{2} Z_{2}\right) B$

$\Omega_{24}=D^{T}\left(h_{1}{ }^{2} Z_{1}+h_{12}{ }^{2} Z_{2}\right)\left(-c N G_{i j}\left(A+A_{\tau}\right)\right.$

$\left.+c l A_{\tau}\right)+c l D^{T}\left(h_{1}^{2} Z_{1}+h_{12}{ }^{2} Z_{2}\right) A_{\tau}$

$\Omega_{33}=B^{T}\left(h_{1}{ }^{2} Z_{1}+h_{12}{ }^{2} Z_{2}\right) B-(1-\mu) R_{3}-K$

$\Omega_{34}=-c N G_{i j} B^{T}\left(h_{1}{ }^{2} Z_{1}+h_{12}{ }^{2} Z_{2}\right)\left(A+A_{\tau}\right)+K \Lambda_{2}$

$+c l B^{T}\left(h_{1}^{2} Z_{1}+h_{12}{ }^{2} Z_{2}\right) A_{\tau}$

$\Omega_{44}=c^{2} N G_{i j}{ }^{2}\left(A+A_{\tau}\right)^{T}\left(h_{1}{ }^{2} Z_{1}+h_{12}{ }^{2} Z_{2}\right)\left(A+A_{\tau}\right)$

$-(1-\mu) Q_{3}-2 Z_{2}-K \Lambda_{1}-c^{2} l N G_{i j}\left(A+A_{\tau}\right)^{T}\left(h_{1}{ }^{2} Z_{1}\right.$

$\left.+h_{12}{ }^{2} Z_{2}\right) A_{\tau}+c^{2} l^{2} A_{\tau}{ }^{T}\left(h_{1}{ }^{2} Z_{1}+h_{12}{ }^{2} Z_{2}\right) A_{\tau}$

$\Omega_{55}=-Z_{1}-Z_{2}-Q_{1}-W \Lambda_{1}$

Then system in (3) is global exponential synchronization.

Proof: We construct the Lyapunov-Krasovskii functional as follows:

$V(x(t))=x^{T}(t)(U \otimes P) x(t)+\int_{t-\tau(t)}^{t} x^{T}(s)\left(U \otimes Q_{3}\right) x(s) d s$ $+\sum_{i=1}^{2} \int_{t-h_{i}}^{t} x^{T}(s)\left(U \otimes Q_{i}\right) x(s) d s+\int_{t-\tau(t)}^{t} f^{T}(x(s))\left(U \otimes R_{3}\right)$ $f(x(s)) d s+\sum_{i=1}^{2} \int_{t-h_{i}}^{t} f^{T}(x(s))\left(U \otimes R_{i}\right) f(x(s)) d s$

$+\int_{-h_{1}}^{0} \int_{t+\theta}^{t} h_{1} \dot{x}^{T}(s)\left(U \otimes Z_{1}\right) \dot{x}(s) d s d \theta$

$+\int_{-h_{2}}^{-h_{1}} \int_{t+\theta}^{t} h_{12} \dot{x}^{T}(s)\left(U \otimes Z_{2}\right) \dot{x}(s) d s d \theta$

Calculating the time derivative of $V(x(t))$ along the trajectories of system (3), from Lemma1 and Lemma2, we have

$\dot{V}(x(t))=2 x^{T}(t)(U \otimes P) \dot{x}(t)+\sum_{i=1}^{3} x^{T}(t)\left(U \otimes Q_{i}\right) x(t)-$ 
$\sum_{i=1}^{2} x^{T}\left(t-h_{i}\right)\left(U \otimes Q_{i}\right) x\left(t-h_{i}\right)-(1-\mu) x^{T}(t-\tau(t))\left(U \otimes Q_{3}\right)$

$x(t-\tau(t))+\sum_{i=1}^{3} f^{T}(x(t))\left(U \otimes R_{i}\right) f(x(t))-\sum_{i=1}^{2} f^{T}\left(x\left(t-h_{i}\right)\right)$

$\left(U \otimes R_{i}\right) f\left(x\left(t-h_{i}\right)\right)-(1-\mu) f^{T}(x(t-\tau(t)))\left(U \otimes R_{3}\right)$

$f(x(t-\tau(t)))+\dot{x}^{T}(t)\left[h_{1}^{2}\left(U \otimes Z_{1}\right)+h_{12}^{2}\left(U \otimes Z_{2}\right)\right] \dot{x}(t)$

$-\int_{t-h_{1}}^{t} h_{1} \dot{x}^{T}(s)\left(U \otimes Z_{1}\right) \dot{x}(s) d s-\int_{t-h_{2}}^{t-h_{1}} h_{12} \dot{x}^{T}(s)\left(U \otimes Z_{2}\right) \dot{x}(s) d s$

Using Lemma 1, we have

$-\int_{t-h_{1}}^{t} h_{1} \dot{x}^{T}(s)\left(U \otimes Z_{1}\right) \dot{x}(s) d s \leq-\left(x(t)-x\left(t-h_{1}\right)\right)^{T}$

$\left(U \otimes Z_{1}\right)\left(x(t)-x\left(t-h_{1}\right)\right)$

On the other hand, based on the approach in [13], and using $h_{12}=\left[h_{2}-\tau(t)\right]+\left[\tau(t)-h_{1}\right]$ we have

$-\int_{t-h_{2}}^{t-h_{1}} h_{12} \dot{x}^{T}(s)\left(U \otimes Z_{2}\right) \dot{x}(s) d s=-\int_{t-h_{2}}^{t-\tau(t)} h_{12} \dot{x}^{T}(s)$

$\left(U \otimes Z_{2}\right) \dot{x}(s) d s-\int_{t-\tau(t)}^{t-h_{1}} h_{12} \dot{x}^{T}(s)\left(U \otimes Z_{2}\right) \dot{x}(s) d s$

$=-\int_{t-h_{2}}^{t-\tau(t)}\left(h_{2}-\tau(t)\right) \dot{x}^{T}(s)\left(U \otimes Z_{2}\right) \dot{x}(s) d s$

$-\int_{t-h_{2}}^{t-\tau(t)}\left(\tau(t)-h_{1}\right) \dot{x}^{T}(s)\left(U \otimes Z_{2}\right) \dot{x}(s) d s$

$-\int_{t-\tau(t)}^{t-h_{1}}\left(\tau(t)-h_{1}\right) \dot{x}^{T}(s)\left(U \otimes Z_{2}\right) \dot{x}(s) d s$

$-\int_{t-\tau(t)}^{t-h_{1}}\left(h_{2}-\tau(t)\right) \dot{x}^{T}(s)\left(U \otimes Z_{2}\right) \dot{x}(s) d s$

Set $\omega=\frac{\tau(t)-h_{1}}{h_{2}}$

$-\int_{t-h_{2}}^{t-\tau(t)}\left(\tau(t)-h_{1}\right) \dot{x}^{T}(s)\left(U \otimes Z_{2}\right) \dot{x}(s) d s$

$=-\omega \int_{t-h_{2}}^{t-\tau(t)} h_{12} \dot{x}^{T}(s)\left(U \otimes Z_{2}\right) \dot{x}(s) d s$

$\leq-\omega \int_{t-h_{2}}^{t-\tau(t)}\left(h_{2}-\tau(t)\right) \dot{x}^{T}(s)\left(U \otimes Z_{2}\right) \dot{x}(s) d s$

$-\int_{t-\tau(t)}^{t-h_{1}} h_{12} \dot{x}^{T}(s)\left(U \otimes Z_{2}\right) \dot{x}(s) d s$

$=-(1-\omega) \int_{t-\tau(t)}^{t-h_{1}} h_{12} \dot{x}^{T}(s)\left(U \otimes Z_{2}\right) \dot{x}(s) d s$

$\leq-(1-\omega) \int_{t-\tau(t)}^{t-h_{1}}\left(\tau(t)-h_{1}\right) \dot{x}^{T}(s)\left(U \otimes Z_{2}\right) \dot{x}(s) d s$

(12)
$W>0, V>0$ and $\Lambda_{1}, \Lambda_{2}$ from assumptions (H3), the following inequality holds

$$
\begin{aligned}
& \sum_{1 \leq i<j \leq N}\left\{-\left[x_{i j}^{T}(t) H \Lambda_{1} x_{i j}(t)-2 x_{i j}^{T}(t) H \Lambda_{2} f\left(x_{i j}(t)\right)+f^{T}\left(x_{i j}(t)\right)\right.\right. \\
& \left.H f\left(x_{i j}(t)\right)\right]-\left[x_{i j}^{T}(t-\tau(t)) K \Lambda_{1} x_{i j}(t-\tau(t))-2 x_{i j}^{T}(t-\tau(t))\right. \\
& \left.K \Lambda_{2} f\left(x_{i j}(t-\tau(t))\right)+f^{T}\left(x_{i j}(t-\tau(t))\right) K f\left(x_{i j}(t-\tau(t))\right)\right]- \\
& {\left[x_{i j}^{T}\left(t-h_{1}\right) W \Lambda_{1} x_{i j}\left(t-h_{1}\right)-2 x_{i j}^{T}\left(t-h_{1}\right) W \Lambda_{2} f\left(x_{i j}\left(t-h_{1}\right)\right)\right.} \\
& \left.+f^{T}\left(x_{i j}\left(t-h_{1}\right)\right) W f\left(x_{i j}\left(t-h_{1}\right)\right)\right]-\left[x_{i j}^{T}\left(t-h_{2}\right) V \Lambda_{1}\right. \\
& x_{i j}\left(t-h_{2}\right)-2 x_{i j}^{T}\left(t-h_{2}\right) V \Lambda_{2} f\left(x_{i j}\left(t-h_{2}\right)\right) \\
& \left.\left.+f^{T}\left(x_{i j}\left(t-h_{2}\right)\right) V f\left(x_{i j}\left(t-h_{2}\right)\right)\right]\right\} \geq 0
\end{aligned}
$$

Adding up (8)-(13) from both sides, we have

$$
\begin{aligned}
& \dot{V}(x(t)) \leq \sum_{1 \leq i<j \leq N} \xi_{i j}^{T}(t) \Phi_{i j} \xi_{i j}(t)-\omega\left[x(t-\tau(t))-x\left(t-h_{2}\right)\right]^{T} \\
& \left(U \otimes Z_{2}\right)\left[x(t-\tau(t))-x\left(t-h_{2}\right)\right]-(1-\omega)\left[x\left(t-h_{1}\right)-x(t-\tau(t))\right]^{T} \\
& \left(U \otimes Z_{2}\right)\left[x\left(t-h_{1}\right)-x(t-\tau(t))\right] \\
& =\sum_{1<i<j \leq N} \xi_{i j}^{T}(t)\left[\Phi_{i j}-\omega I_{1}^{T} Z_{2} I_{1}-(1-\omega) I_{2}{ }^{T} Z_{2} I_{2} \xi_{i j}(t)\right. \\
& =\sum_{i=1}^{N-1} \sum_{j=i+1}^{N} \xi_{i j}(t)^{T}\left[(1-\omega) \Phi_{i j}^{1}+\omega \Phi^{2}{ }_{i j} \xi_{i j}(t)\right.
\end{aligned}
$$

where $\phi_{i j} \phi_{i j}{ }^{1} \phi_{i j}{ }^{2}$ are given in(17), (18) and (19) respectively, and $I_{1}=\left[\begin{array}{lllll}0_{n, 3 n} & -I_{n} & 0_{n} & -I_{n} & 0_{n, 2 n}\end{array}\right]$

$$
\begin{aligned}
& I_{2}=\left[\begin{array}{llll}
0_{n, 3 n} & -I_{n} & I_{n} & 0_{n, 3 n}
\end{array}\right] . \\
& \xi_{i j}{ }^{T}(t)=\left[x _ { i j } { } ^ { T } ( t ) \quad f ^ { T } ( x _ { i j } ( t ) ) \quad f ^ { T } \left(x_{i j}(t-\tau(t)) \quad x_{i j}{ }^{T}(t-\tau(t))\right.\right. \\
& \left.x_{i j}{ }^{T}\left(t-h_{1}\right) \quad x_{i j}{ }^{T}\left(t-h_{2}\right) \quad f^{T}\left(x_{i j}\left(t-h_{1}\right)\right) \quad f^{T}\left(x_{i j}\left(t-h_{2}\right)\right)\right] .
\end{aligned}
$$

Following theorm2, we have $(1-\omega) \Phi^{1}{ }_{i j}+\omega \Phi^{2}{ }_{i j}<0$, and exist a positive constant $M>0$ satisfied $(1-\omega) \Phi^{1}{ }_{i j}+\omega \Phi^{2}{ }_{i j} \leq-M I<0$. Such that

$$
\begin{gathered}
\dot{V}(x(t)) \leq \sum_{i=1}^{N-1} \sum_{j=i+1}^{N} \xi_{i j}(t)^{T}\left[(1-\omega) \Phi^{1}{ }_{i j}+\omega \Phi^{2}{ }_{i j}\right] \xi_{i j}(t) \\
\leq-M \sum_{i=1}^{N-1} \sum_{j}^{N}\left[\left\|x_{i j}(t)\right\|^{2}+\left\|x_{i j}(t-\tau(t))\right\|^{2}\right]
\end{gathered}
$$

Furthermore, based on the proof in [9], there exist two positive scalars $\beta>0, k>0$ such that

For any $n \times n$ diagonal matrices $H>0, K>0$, 
$\left\|x_{i j}(t)\right\| \leq \beta \sum_{i=1}^{N-1} \sum_{j=i+1}^{N} \sup _{-2 h_{2} \leq s \leq 0}\left\|\phi_{i}(s)-\phi_{j}(s)\right\| \cdot e^{-k t}$

for $t \geq \boldsymbol{T}$.By Definition 1, therefore system (3) is global exponential synchronization. Then end the proof.

Remark 2. In [6-9], the authors studied the synchronization of an array of linearly coupled networks with constant coupling delay or time-varying coupling delay, and the derivative of the time-varying delay is confined to be less than 1 . We remove this restrictive and the derivative of the time-varying delay can be any value.

Remark 3: During the estimation, $--_{t-h_{2}}^{t-h_{1}} h_{12} \dot{x}^{T}(s)\left(U \otimes Z_{2}\right) \dot{x}(s) d s$ is separated to two parts as follows $-\int_{t-h_{2}}^{t-h_{1}} h_{12} \dot{x}^{T}(s)\left(U \otimes Z_{2}\right) \dot{x}(s) d s=-\int_{t-h_{2}}^{t-\tau(t)} h_{12} \dot{x}^{T}(s)\left(U \otimes Z_{2}\right)$ $\dot{x}(s) d s-\int_{t-\tau(t)}^{t-h_{1}} h_{12} \dot{x}^{T}(s)\left(U \otimes Z_{2}\right) \dot{x}(s) d s \quad$ and $\quad$ we estimate each part respectively, ignoring the direct estimate for $-\int_{t-h_{2}}^{t-h_{1}} h_{12} \dot{x}^{T}(s)\left(U \otimes Z_{2}\right) \dot{x}(s) d s$, then we have more accurate estimate than those from the reference[7-9].

\section{NUMERICAL EXAMPLES}

In the section, a examples is provided to illustrate the effectiveness of the conclusion.

Example1: We consider the following DNNs

$$
\dot{x}_{i}(t)=-C x_{i}(t)+D f\left(x_{i}(t)\right)+B f\left(x_{i}(t-\tau)\right)+I(t)
$$

Let us see the synchronized states of a chaotic system: Chua's circuit.

The dynamics of Chua's circuit is

$$
\left\{\begin{array}{l}
\frac{d x_{1}}{d t}=10\left(-x_{1}+x_{2}-f\left(x_{1}\right)\right) \\
\frac{d x_{2}}{d t}=x_{1}-x_{2}+x_{3} \\
\frac{d x_{3}}{d t}=-18 x_{2}
\end{array}\right.
$$

where

$$
\begin{aligned}
& f\left(x_{1}\right)=b x_{1}+0.5(d-b)\left(\left|x_{1}+1\right|-\left|x_{1}-1\right|\right), \\
& d=-4 / 3, b=-3 / 4 .
\end{aligned}
$$

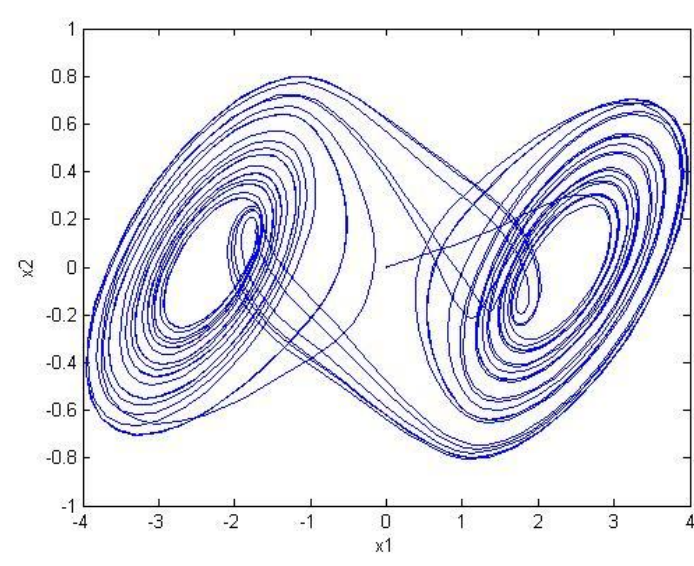

Fig.1 illustrates the chaotic trajectories of system (16).

Here we choose Chua's circuit (16) as the uncoupled system. The coupling time delay as $\tau(t)=0.03\left(2+\sin (40 t)+\cos ^{2}(80 t)\right) \quad$ and $\quad$ the coupling strength $c=1$, then we consider a dynamic networks consisting of three linearly coupled identical DNNs with couplings as

$\dot{x}_{i}(t)=-C x_{i}(t)+D f\left(x_{i}(t)\right)+B f\left(x_{i}(t-\tau(t))\right)+I(t)+c \sum_{j=1, j \neq i}^{N} G_{i j} A\left[x_{j}(t\right.$
$\left.-\tau(t))-x_{i}(t-\tau(t))\right]+c \sum_{j=1 j \neq i}^{N} G_{i j} A_{\tau}\left[x_{j}(t-\tau)-x_{i}(t)\right]$

for $i=1,2,3$. Let

$C=\left[\begin{array}{ccc}10 & -10 & 0 \\ -1 & 1 & -1 \\ 0 & 18 & 0\end{array}\right]$ and $D=\left[\begin{array}{ccc}-10 & 0 & 0 \\ 0 & 0 & 0 \\ 0 & 0 & 0\end{array}\right]$,

$G=\left[\begin{array}{ccc}-4 & 2 & 2 \\ 2 & -4 & 2 \\ 2 & 2 & -4\end{array}\right], A=\left[\begin{array}{lll}1 & 0 & 0 \\ 0 & 1 & 0 \\ 0 & 0 & 1\end{array}\right]$

$A_{\tau}=\left[\begin{array}{ccc}0.5 & 0.1 & 0 \\ 0 & 0.5 & 0 \\ 0.3 & 0 & 0.5\end{array}\right], I(t)=B=0$, and $N=3, l=4$.

By calculating we have the activation functions $f\left(x_{i}\right)$ satisfy Assumption H3. If the system reaches synchronization, we have the following synchronized state equation:

$\dot{s}(t)=-C s(t)+D f(s(t))+B f(s(t-\tau))+l A_{\tau}(s(t-\tau)-s(t))$.

By calculating other variables in theorem 1 Using Matlab Toolbox, we realize the system synchronization and the total error is given as 
$\operatorname{error}(t)=\sum_{i=1}^{3} \sqrt{\left[x_{1 i}-x_{2 i}\right]^{2}+\left[x_{2 i}-x_{3 i}\right]^{2}} \quad$ Fig.2 shows the synchronized state of system (17) and the synchronous error with the initial value randomly chosen from $[0,1]$.
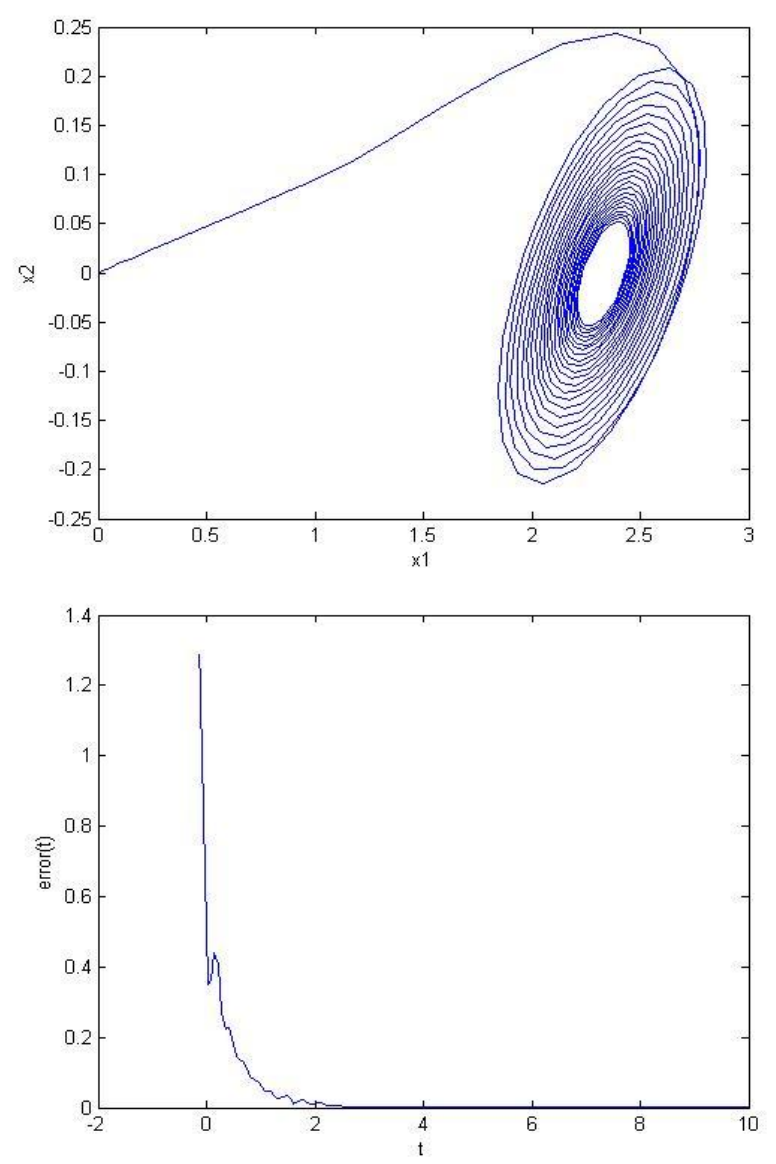

Fig.2 the synchronized state of system (17) and the synchronous error.

\section{CONCLUSIONS}

In this paper, global synchronization problem is investigated for a general complex networks with coupling delays. A novel theorem is obtained about synchronization of the coupled systems by employing Lyapunov-Krasovskii functional. The condition obtained in this paper is expressed in the form of linear matrix inequalities, which has less variables and easy to be computed and checked by resorting to Matlab LMI Toolbox. The proposed network model may shed some new lights on the synchronization with one delay coupling. Furthermore, there are abundant dynamical behaviors in arrays of coupled systems with different coupling schemes and it deserves to be further studied in future. Finally, the example is utilized to illustrate the usefulness of the derived methods by simulation results.

\section{References}

1. Wang J, Xu Z New study on neural networks: the essential order of approximation. Neural Netw 23:618-624, 2010.

2. Wang G, Cao J, Wang L, Franklin J. Global dissipativity of stochastic neural networks with time delay. J Franklin Inst 346:794-807, 2009.

3. Trestian I, Ranjan S, Kuzmanovic A, Nucci A, Googling the internet: profiling internet endpoints via the World Wide Web. IEEE Trans Neural Netw 18:666-679, 2010.

4. Yuan ZZ, Cai JP, and Lin ML. Global synchronization in complex networks with adaptive coupling. Mathematical Problems in Engineering, Article ID 826721, 2010.

5. Lü JH, Chen GR. A time-varying complex dynamical network model and its controlled synchronization criteria. IEEE Transactions on Automatic Control, 50:841-846,2005.

6. Li CG, Chen GR. Synchronization in general complex delayed dynamical networks with coupling delays. Physica A 343:263-278, 2004.

7. Gao HJ, Lam J, Chen GR. New criteria for synchronization stability of general complex dynamical networks with coupling delays .Phys. Lett. A 360:263273, 2006.

8. Li K, Guan SG, Gong XF. Synchronization stability of general complex dynamical networks with time-varying delays. Phys. Lett. A 372: $713-727$, 2008.

9. Li HJ, Yue D. Synchronization stability of general complex dynamical networks with time-varying delays: A piecewise analysis method. J Comput Appl Math 232:149-158, 2009.

10. Song QK Synchronization analysis of coupled connected neural networks with mixed time delays Neurocomputing, vol. 72, no. 16-18, pp. 3907-3914, 2009.

11. Wang Y, Wang ZD, Liang JL. A delay fractioning approach to global synchronization of delayed complex networks with stochastic disturbances. Phys Letters A 372:6066-6073, 2008.

12. Shao HY. New delay-dependent stability criteria for systems with interval delay. Automatica, vol. 45, no. 3, pp. 744-749, 2009.

13. Li T, Wang T. Exponential Synchronization for Arrays of Coupled Neural Networks with Time-delay Couplings. J Control, Automation, 9(1):187-196, 2011. 